\title{
RELATIVE PÓLYA GROUP AND PÓLYA DIHEDRAL EXTENSIONS OF $\mathbb{Q}$
}

\author{
ABBAS MAAREFPARVAR AND ALI RAJAEI*
}

\begin{abstract}
A number field with trivial Pólya group 2 is called a Pólya field. We define "relative Pólya group $\operatorname{Po}(L / K)$ " for $L / K$ a finite extension of number fields, generalizing the Pólya group. Using cohomological tools in [1, we compute some relative Pólya groups. As a consequence, we generalize Leriche's results in [17] and prove the triviality of relative Pólya group for the Hilbert class field of $K$. Then we generalize our previous results [19] on Pólya $S_{3}$ extensions of $\mathbb{Q}$ to dihedral extensions of $\mathbb{Q}$ of order $2 l$, for $l$ an odd prime. We also improve Leriche's upper bound in [16] on the number of ramified primes in Pólya $D_{l}$-extensions of $\mathbb{Q}$ and prove that for a real (resp. imaginary) Pólya $D_{l}$-extension of $\mathbb{Q}$ at most 4 (resp. 2) primes ramify.
\end{abstract}

Keywords: integer valued polynomial, Pólya field, Pólya group, relative Pólya group, dihedral extension.

Notation. The following notation will be used throughout this article:

For a number field $K, I(K), P(K), \mathrm{Cl}(K), \mathcal{O}_{K}, h(K), U_{K}, w_{K}, H(K), \Gamma(K)$ and $D_{K}$ denote the group of fractional ideals, group of principal fractional ideals, ideal class group, ring of integers, class number, group of units, Dirichlet rank of group of units, Hilbert class field, genus field and discriminant of $K$, respectively.

For a finite extension $L / K$ of number fields, $\mathcal{N}_{L / K}: \mathrm{Cl}(L) \rightarrow \mathrm{Cl}(K)$ denotes the induced morphism by the ideal norm morphism $N_{L / K}: I(L) \rightarrow I(K)$. Likewise $\epsilon_{L / K}: \mathrm{Cl}(K) \rightarrow \mathrm{Cl}(L)$ denotes the transfer of ideal classes induced by the morphism $j_{L / K}: \mathfrak{a} \in I(K) \mapsto \mathfrak{a} \mathcal{O}_{L} \in I(L)$.

For a prime ideal $\mathfrak{p}$ of $K$ and a prime ideal $\mathfrak{B}$ of $L$ above $\mathfrak{p}$, denote the ramification index and residue class degree of $\mathfrak{B}$ over $\mathfrak{p}$ by $e(\mathfrak{B} / \mathfrak{p})$ and $f(\mathfrak{B} / \mathfrak{p})$, respectively.

Finally, $l$ is an odd prime number, and for integer $n \geq 3, C_{n}$ and $D_{n}$ denote the cyclic group of order $n$ and the dihedral group of order $2 n$, respectively.

\section{INTRODUCTION}

Historically, the study of Pólya fields dates back to Pólya's results on integer valued polynomials 22. For a number field $K$, with ring of integers $\mathcal{O}_{K}$, the ring of integer valued polynomials on $\mathcal{O}_{K}$ is defined as follows:

$$
\operatorname{Int}\left(\mathcal{O}_{K}\right)=\left\{f \in K[x] \mid f\left(\mathcal{O}_{K}\right) \subseteq \mathcal{O}_{K}\right\} .
$$

One can show that $\operatorname{Int}\left(\mathcal{O}_{K}\right)$ is free as an $\mathcal{O}_{K}$-module, but an explicit $\mathcal{O}_{K}$-basis for $\operatorname{Int}\left(\mathcal{O}_{K}\right)$ may be difficult to write down, and Pólya [22] was interested in those fields $K$ for which $\operatorname{Int}\left(\mathcal{O}_{K}\right)$ has an $\mathcal{O}_{K}$ basis which exactly one member from each degree. Such basis, if it exists, is called a regular basis.

2010 Mathematics Subject Classification. Primary 11R04, 11R29, 11R34, 11R37, 13F20.

${ }^{*}$ Corresponding author. 
For a nonnegative integer $n$, denote the subset of $K$ formed by 0 and the leading coefficients of integer valued polynomials of degree $n$ on $\mathcal{O}_{K}$ by $\mathfrak{J}_{n}(K)$. This is a fractional ideal of $\mathcal{O}_{K}: \mathfrak{J}_{n}(K) \subseteq(n !)^{-1} \mathcal{O}_{K}$, see [24, Section 2]. Pólya proved that $\operatorname{Int}\left(\mathcal{O}_{K}\right)$ has a regular basis if and only if all the ideals $\mathfrak{J}_{n}(K)$ are principal, see 22, Satz I]. Immediately after Pólya, Ostrowski 21] proved that $\operatorname{Int}\left(\mathcal{O}_{K}\right)$ has a regular basis if and only if all the ideals

$$
\Pi_{q}(K)=: \prod_{\substack{\mathfrak{m} \in \operatorname{Max}\left(\mathcal{O}_{K}\right) \\ N_{K / \mathbb{Q}}(\mathfrak{m})=q}} \mathfrak{m}
$$

are principal.

Note that for a Galois number field $K$, existence of a regular basis for $\operatorname{Int}\left(\mathcal{O}_{K}\right)$ is equivalent to principality of $\prod_{i=1}^{g} \mathfrak{P}_{i}$, where $\mathfrak{P}_{1}, \mathfrak{P}_{2}, \ldots, \mathfrak{P}_{g}$ are all distinct prime ideals of $K$ above a ramified prime $p$, see [24, Section 1].

Definition 1.1. 24] A number field $K$ is called Pólya, if the $\mathcal{O}_{K}$-module $\operatorname{Int}\left(\mathcal{O}_{K}\right)$ admits a regular basis.

Definition 1.2. 2, Definition II.3.8] For a number field $K$, the Pólya-Ostrowski group or Pólya group of $K$ is the subgroup $\mathrm{Po}(K)$ of $\mathrm{Cl}(K)$ generated by the classes of the ideals $\mathfrak{J}_{n}(K)$. It is easy to see that this is same as the subgroup generated by the classes of the ideals $\Pi_{q}(K)$ as well, see [2, Proposition II.3.9].

Now let $K$ be a Galois extension of $\mathbb{Q}$ with Galois group $G$. One can show that the Ostrowski ideals $\Pi_{q}(K)$ freely generate the ambiguous ideals $I(K)^{G}$, see 1. Section 2]. Thus $\operatorname{Po}(K)$ is the subgroup of $\mathrm{Cl}(K)$ generated by the classes of ambiguous ideals. For quadratic fields, Hilbert proved:

Proposition 1.3. 10. Theorem 106] Let $K$ be a quadratic field and denote the number of ramifid primes in $K / \mathbb{Q}$ by $s_{K}$. If $K$ is real and the fundamental unit of $K$ has positive norm, then $\# \operatorname{Po}(K)=2^{s_{k}-2}$. Otherwise $\# \operatorname{Po}(K)=2^{s_{k}-1}$.

The above theorem of Hilbert has been generalized by Zantema:

Proposition 1.4. 24, Section 3, page 9] Let $K / \mathbb{Q}$ be a Galois extension with Galois group $G$. Denote the ramification index of a prime $p$ in $K$ by $e_{p}$. Then the following sequence is exact:

$$
\{0\} \longrightarrow H^{1}\left(G, U_{K}\right) \longrightarrow \bigoplus_{p \text { prime }} \mathbb{Z} / e_{p} \mathbb{Z} \longrightarrow \operatorname{Po}(K) \longrightarrow\{0\} .
$$

Remark 1.5. By Zantema's exact sequence (1.1), for a Galois number field $K$, \# $\operatorname{Po}(K)$ divides $\prod_{p \text { prime }} e_{p}$, so if $\operatorname{gcd}([K: \mathbb{Q}], h(K))=1$ then $K$ is Pólya, but not conversely. For instance, as we will see, in Example (3.13) there is a Pólya $D_{7}$-extension of $\mathbb{Q}$ with class number 7 .

The reader is refered to $[2,3,4,15,8,9,15,16,17,18,19,24$, for some results on Pólya fields and Pólya groups.

\section{Relative Pólya group}

Using Brumer-Rosen's method in 1], we can generalize Zantema's result in [24, Section 3] to finite Galois extensions of number fields and find a generalization of exact sequence (1.1). First we recall the definition of the relative Pólya group [18] (also independently defined by Chabert [4]): 
Definition 2.1. [18, Definition 4.1] Let $L / K$ be a finite extension of number fields. The relative Pólya group of $L$ over $K$, is the subgroup of $\mathrm{Cl}(L)$ generated by the classes of the ideals $\Pi_{\mathfrak{P} f}(L / K)$, where $\mathfrak{P}$ is a prime ideal of $K, f$ is a positive integer and $\Pi_{\mathfrak{P}^{f}}(L / K)$ is defined as follows:

$$
\Pi_{\mathfrak{P}^{f}}(L / K)=\prod_{\substack{\mathfrak{M} \in \operatorname{Max}\left(\mathcal{O}_{L}\right) \\ N_{L / K}(\mathfrak{M})=\mathfrak{P}^{f}}} \mathfrak{M}
$$

We denote the relative Pólya group of $L$ over $K$ by $\operatorname{Po}(L / K)$. In particular, $\operatorname{Po}(L / \mathbb{Q})=\operatorname{Po}(L)$ and $\operatorname{Po}(L / L)=\mathrm{Cl}(L)$.

Now let $L / K$ be a Galois extension with Galois group $G$. It is easily seen that the set of all ideals $\Pi_{\mathfrak{P}^{f}}(L / K)$ is a set of free generators for the ambiguous ideals $I(L)^{G}$, see [1, Proof of Proposition 2.2]. On the other hand $P(L)^{G}=I(L)^{G} \cap P(L)$. Hence in this case $\operatorname{Po}(L / K)=I(L)^{G} / P(L)^{G}$. As in [24, Section 3], we define:

$$
\begin{array}{r}
\psi: I(L)^{G} \rightarrow \bigoplus_{\mathfrak{P} \text { is a prime of } \mathrm{K}} \mathbb{Z} / e_{\mathfrak{P}} \mathbb{Z} \\
\left(\psi\left(\prod_{i=1}^{m} \Pi_{\mathfrak{P}^{f_{i}}}(L / K)^{t_{i}}\right)\right)_{\mathfrak{P}_{\mathfrak{i}}}:=t_{i}\left(\bmod e_{\mathfrak{P}_{\mathfrak{i}}}\right),
\end{array}
$$

where $m$ is a positive integer, $t_{i}$ 's are integers and $e_{\mathfrak{P}}$ denotes the ramification index of $\mathfrak{P}$ in $L / K$. It is clear that $\psi$ is a group epimorphism and one can show that $\operatorname{Ker}(\psi)=I(K)$. Hence we get the following exact sequence:

$$
\{0\} \longrightarrow I(K) \longrightarrow I(L)^{G} \longrightarrow \underset{\mathfrak{P} \text { is prime of } \mathrm{Z}}{\bigoplus} \mathbb{Z} / e_{\mathfrak{P}} \mathbb{Z} \longrightarrow\{0\}
$$

Following Brumer-Rosen [1, Consider the following exact sequence:

$$
\{0\} \longrightarrow U_{L} \longrightarrow L^{*} \longrightarrow P(L) \longrightarrow\{0\} .
$$

Taking Cohomology and using Hilbert's theorem 90 [10], we get the exact sequence

$$
\{0\} \longrightarrow U_{K} \longrightarrow K^{*} \longrightarrow P(L)^{G} \longrightarrow H^{1}\left(G, U_{L}\right) \longrightarrow\{0\}
$$

Equivalently, the following sequence is exact:

$$
\{0\} \longrightarrow P(K) \longrightarrow P(L)^{G} \longrightarrow H^{1}\left(G, U_{L}\right) \longrightarrow\{0\} .
$$

Now we can generalize Zantema's result in [24, Section 3]:

Theorem 2.2. Let $L / K$ be a finite Galois extension of number fields with Galois group $G$. Then the following sequence is exact:

$$
\{0\} \rightarrow \operatorname{Ker}\left(\epsilon_{L / K}\right) \rightarrow H^{1}\left(G, U_{L}\right) \rightarrow \bigoplus_{\mathfrak{P} \text { is prime of } K} \mathbb{Z} / e_{\mathfrak{P}} \mathbb{Z} \rightarrow \frac{\operatorname{Po}(L / K)}{\epsilon_{L / K}(\mathrm{Cl}(K))} \rightarrow\{0\} .
$$


Proof. We have the following commutative diagram with exact rows of abelian groups:

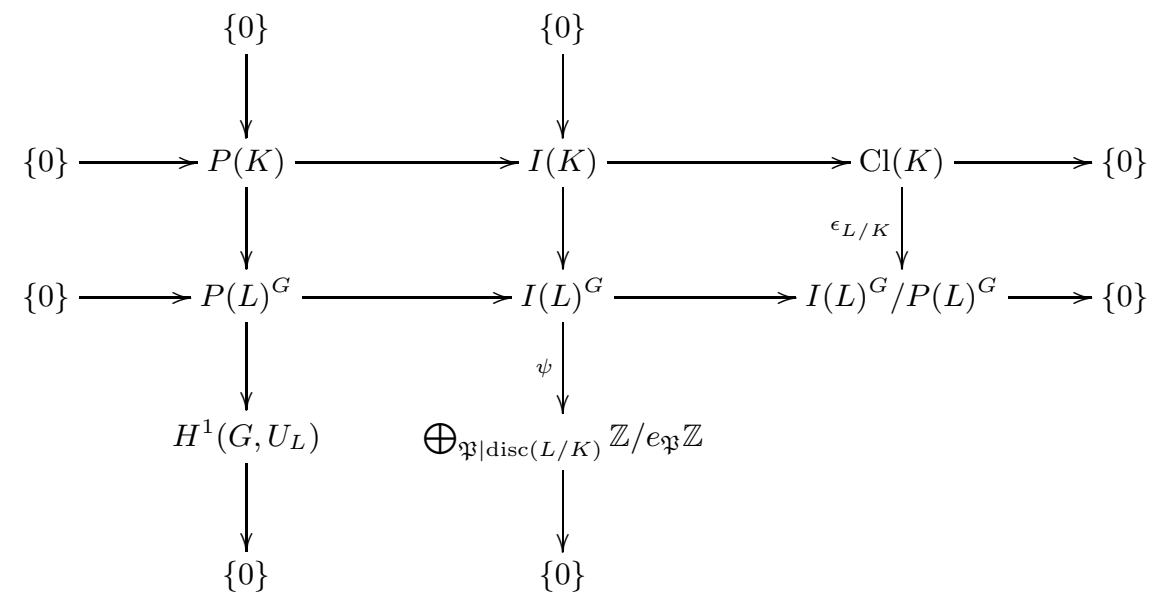

The first column is the exact sequence (2.3), and the second column is the exact sequence (2.2). Hence by the snake lemma, we find an exact sequence as follows:

$$
\{0\} \rightarrow \operatorname{Ker}\left(\epsilon_{L / K}\right) \rightarrow H^{1}\left(G, U_{L}\right) \rightarrow \bigoplus_{\mathfrak{P} \mid \operatorname{disc}(L / K)} \mathbb{Z} / e_{\mathfrak{P}} \mathbb{Z} \rightarrow \operatorname{Coker}\left(\epsilon_{L / K}\right) \rightarrow\{0\}
$$

Since $L / K$ is a Galois extension, $\operatorname{Po}(L / K)=I(L)^{G} / P(L)^{G}$ and the statement is proved.

Remark 2.3. By exact sequence (2.4), order of the quotient group $\frac{\operatorname{Po}(L / K)}{\epsilon_{L / K}(\mathrm{Cl}(K))}$ divides a power of $[L: K]$. On the other hand, $\operatorname{Po}(L / K)$ is a subgroup of $\operatorname{Cl}(L)$. Hence if $\operatorname{gcd}(h(L),[L: K])=1$, then $\operatorname{Po}(L / K)=\epsilon_{L / K}(\mathrm{Cl}(K))$.

Corollary 2.4. Let $L / K$ be a finite Galois extension of number fields with Galois group $G$. If $h(K)$ is relatively prime to $[L: K]$, then $\mathrm{Cl}(K)$ is embedded in $\operatorname{Po}(L / K)$. In particular, $h(K)$ divides $h(L)$. Moreover, we get a generalization of Zantema's exact sequence (1.1) as follows:

$$
\{0\} \rightarrow H^{1}\left(G, U_{L}\right) \rightarrow \bigoplus_{\mathfrak{P} \mid \operatorname{disc}(L / K)} \mathbb{Z} / e_{\mathfrak{P}} \mathbb{Z} \rightarrow \operatorname{Po}(L / K) / \mathrm{Cl}(K) \rightarrow\{0\} .
$$

Proof. Since $\operatorname{gcd}(h(K),[L: K])=1, \mathcal{N}_{L / K} o \epsilon_{L / K}: \overline{\mathfrak{a}} \in \mathrm{Cl}(K) \mapsto \overline{\mathfrak{a}}^{[L: K]} \in \mathrm{Cl}(K)$ is injective. Hence $\epsilon_{L / K}$ is injective and the statement follows from Theorem (2.2).

Remark 2.5. By the above proof, if $\operatorname{gcd}(h(K),[L: K])=1$, then $\epsilon_{L / K}$ is injective, even for non-Galois extensions $L / K$. Indeed this is a particular case of Leriche's result in [16, Proposition 6.3].

Remark 2.6. If we are just interested in $\# \mathrm{Po}(L / K)$ of a Galois extensions $L / K$ (without the hypothesis that $h(K)$ and $[L: K]$ are coprime), then interchanging the horizontal and vertical rows in the diagram in proof of Theorem (2.2), gives

$$
\# \operatorname{Po}(L / K)=\frac{h(K) \cdot \prod_{\mathfrak{P} \mid \operatorname{disc}(L / K)} e_{\mathfrak{P}}}{\# H^{1}\left(G, U_{L}\right)} .
$$

This has been done in [4, Proposition 4.4]. 
Corollary 2.7. Let $L / K$ be a finite Galois extension of number fields with Galois group $G$. If every ideal class of $K$ extended to $L$ is principal, then

$$
\{0\} \rightarrow \mathrm{Cl}(K) \rightarrow H^{1}\left(G, U_{L}\right) \rightarrow \bigoplus_{\mathfrak{P} \text { is prime of } K} \mathbb{Z} / e_{\mathfrak{P}} \mathbb{Z} \rightarrow \operatorname{Po}(L / K) \rightarrow\{0\}
$$

is exact.

Proof. In this case, $\operatorname{Ker}\left(\epsilon_{L / K}\right)=\mathrm{Cl}(K)$ and the statement follows from Theorem (2.2). Note that this generalizes Zantema's exact sequence (1.1).

Corollary 2.8. Let $L / K$ be a finite Galois extension of number fields with Galois group $G$. If all finite places of $K$ are unramified in $L$, then $H^{1}\left(G, U_{L}\right)$ is embedded in $\mathrm{Cl}(K)$ and $\mathrm{Po}(L / K) \simeq \epsilon_{L / K}(\mathrm{Cl}(K))$.

Proof. If all finite places of $K$ are unramified in $L$, by exact sequence (2.4), we have $H^{1}\left(G, U_{L}\right) \simeq \operatorname{Ker}\left(\epsilon_{L / K}\right)$ is a subgroup of $C l(K)$ and the quotient group $\frac{\operatorname{Po}(L / K)}{\epsilon_{L / K}(\operatorname{Cl}(K))}$ would be trivial .

Corollary 2.9. Let $L / K$ be a finite Galois extension of number fields with Galois group $G$. If every ideal class of $K$ extended to $L$ is principal and all finite places of $K$ are unramified in $L$, then $\mathrm{Cl}(K) \simeq H^{1}\left(G, U_{L}\right)$ and $\operatorname{Po}(L / K)=\{0\}$. In particular, $\operatorname{Po}(H(K) / K)$ is trivial, for the Hilbert class field $H(K)$ of $K$.

Proof. Immediately follows from Corollaries (2.7) and (2.8).

Leriche [17 proved that the Hilbert class field $H(K)$ of $K$ is a Pólya field. Using the following lemma (part $(i i)$, for $P=\mathbb{Q}$ ), we conclude that Corollary (2.9) is a generalization of this result [17, Corollary 3.2]:

Lemma 2.10. Let $P \subseteq K \subseteq L$ be a tower of finite extensions of number fields.

(i) If $K / P$ and $L / P$ are Galois extensions, then $\epsilon_{L / K}(\operatorname{Po}(K / P)) \subseteq \operatorname{Po}(L / P)$.

(ii) If $L / K$ is a Galois extension, then $\operatorname{Po}(L / P) \subseteq \operatorname{Po}(L / K)$.

Proof. (i) Let $\mathfrak{p}, \beta$ and $\gamma$ be respectively primes of $P, K$ and $L$ such that $\gamma|\beta| \mathfrak{p}$. Since $K / P$ and $L / P$ are Galois extensions, one has

$$
j_{L / K}\left(\Pi_{\mathfrak{p}^{f}}(K / P)\right)=\left(\Pi_{\mathfrak{p} f^{\prime}}(L / P)\right)^{e(\gamma / \beta)} \in \operatorname{Po}(L / P),
$$

where $f=f(\beta / \mathfrak{p})$ and $f^{\prime}=f(\gamma / \mathfrak{p})$.

(ii) Let

$$
\begin{aligned}
\mathfrak{p} \mathcal{O}_{K} & =\beta_{1}^{e_{1}} \beta_{2}^{e_{2}} \ldots \beta_{g}^{e_{g}}, \\
\mathfrak{p O} \mathcal{O}_{L} & =\gamma_{1}^{e_{1}^{\prime}} \gamma_{2}^{e_{2}^{\prime}} \ldots \gamma_{t}^{e_{t}^{\prime}},
\end{aligned}
$$

be the decomposition forms of $\mathfrak{p}$ in $K / P$ and $L / P$, respectively. For a positive integer $f$, let $\Pi_{\mathfrak{p}^{f}}(L / P)=\prod_{j=1}^{d} \gamma_{j}$, and $\left\{\beta_{1}, \beta_{2}, \ldots, \beta_{s}\right\}=\left\{\gamma_{j} \cap K: j=1,2, \ldots, d\right\}$ be set of the all distinct prime ideals of $K$ below $\gamma_{j}$ 's. Also for every $i=1, \ldots, s$, let $\left\{\gamma_{i, 1}, \ldots, \gamma_{i, u_{i}}\right\}$ be the set of the all distinct prime ideals of $L$ above $\beta_{i}$. Since $L / K$ is a Galois extension, for every $i=1, \ldots, s$, the ideals $\gamma_{i, 1}, \gamma_{i, 2}, \ldots, \gamma_{i, u_{i}}$ have the same ideal norm (over $K$ ), say $\beta_{i}^{\mathfrak{f}_{i}}$. Hence

$$
\Pi_{\mathfrak{p}^{f}}(L / P)=\prod_{i=1}^{s} \Pi_{\beta_{i}^{\mathrm{f}_{i}}}(L / K) \in \operatorname{Po}(L / K) .
$$


Remark 2.11. For a finite extension $L / K$ of Galois number fields, Chabert proved that $\epsilon_{L / K}(\operatorname{Po}(K)) \subseteq \operatorname{Po}(L)$, see [3, Proposition 3.4]; part $(i)$ in Lemma (2.10) is a relativization of this result.

Remark 2.12. Note that if either $K / P$ or $L / P$ is not Galois, then the containment in the part $(i)$ in Lemma (2.10) might not hold. For instance, consider the pure cubic field $K=\mathbb{Q}(\sqrt[3]{19})$. One can show that the Galois closure $L$ of $K$ is a Pólya field while $\operatorname{Po}(K)=\mathrm{Cl}(K) \simeq \mathbb{Z} / 3 \mathbb{Z}$, see [19, Example 2.9]. On the other hand since $\operatorname{gcd}(h(K),[L: K])=1, \mathcal{N}_{L / K} o \epsilon_{L / K}: \overline{\mathfrak{a}} \in \mathrm{Cl}(K) \mapsto \overline{\mathfrak{a}}^{[L: K]} \in \mathrm{Cl}(K)$ is injective, so is $\epsilon_{L / K}$. Therefore $\epsilon_{L / K}(\mathrm{Po}(K))=\mathrm{Po}(K)=\mathrm{Cl}(K) \nsubseteq \mathbb{P o}(L)$.

Remark 2.13. By Corollary (2.9) and Lemma (2.10), $\operatorname{Po}(H(H(K)) / K)=\{0\}$. Hence every number field in the tower of Hilbert class fields for $K$ has trivial relative Pólya group over $K$.

Leriche also proved that the genus field $\Gamma(K)$ of an abelian number field $K$ is Pólya, see [17, Theorem 3.8]. But unlike the Hilbert class field, the relative Pólya group of the genus field, even for abelian number fields, is not necessarily trivial:

Example 2.14. For $K=\mathbb{Q}(\sqrt{-23})$, we have $h(K)=3$. By [11, Lemma 3], the Hilbert class field $H(K)$ is a Galois extension of $\mathbb{Q}$. Since $H(K) / K$ is unramified, one can easily show that $H(K) / \mathbb{Q}$ cannot be cyclic (see proof of Lemma (3.5)), so $\operatorname{Gal}(H(K) / \mathbb{Q}) \simeq S_{3}$. On the other hand any compositum of $K$ and a cyclic cubic extension of $\mathbb{Q}$ is abelian. Thus $\Gamma(K)=K$, and $\operatorname{Po}(\Gamma(K) / K)=\mathrm{Cl}(K) \simeq \mathbb{Z} / 3 \mathbb{Z}$.

\section{Main Result}

From now on, $K$ is a non-Galois number field of degree $l$ (an odd prime) whose Galois closure has Galois group isomorphic to $D_{l}$, the dihedral group of odrer $2 l$. Denote the Galois closure of $K$ by $L$ and the unique quadratic subfield of $L$ by $E$.

Suppose that $p$ is a ramified prime in $L$, with decomposition in $L$ by $p \mathcal{O}_{L}=$ $\left(\gamma_{1} \gamma_{2} \ldots \gamma_{g}\right)^{e(p)}$. Since $e(p) f(p) g=[L: \mathbb{Q}]=2 l$, we have $e(p)=2$ or $e(p)=l$ or $e(p)=2 l$, where $f(p)$ is the residue class degree of $\gamma_{i}$ 's over $p$.

With these notations, following [7] we restate the complete description of decomposition forms of ramified primes $p$ in $K$ and $L$ :

Proposition 3.1. With $K$ and $L$ as above:

(1) If $e(p)=2$, then $f(p)=1$. Moreover, if

$$
p \mathcal{O}_{L}=\gamma_{1}^{2} \gamma_{2}^{2} \ldots \gamma_{l}^{2}
$$

is the decomposition of $p$ in $L$, then the decomposition of $p$ in $K$ has the form below:

$$
p \mathcal{O}_{K}=\beta_{1} \beta_{2}^{2} \ldots \beta_{\frac{l+1}{2}}^{2} .
$$

(2) If $e(p)=l$, then $f(p)=1$ or $f(p)=2$ and $p$ is totally ramified in $K$.

(3) If $e(p)=2 l$, then $p=l$ and it is totally ramified in $K$.

Proof. A detailed analysis of ramification groups using [23, Chapter III] yields the claims, for details see [7, Proposition 10.1.26].

Theorem 3.2. With $E, K$, and $L$ as above, we have an exact sequence as follows:

$$
\{0\} \longrightarrow \mathrm{Po}(E) \longrightarrow \mathrm{Po}(L) \longrightarrow \mathrm{Po}(K) \text {. }
$$


Moreover, the 2-torsion subgroup of $\operatorname{Po}(L)$ is isomorphic to $\operatorname{Po}(E)$ and the l-torsion subgroup of $\operatorname{Po}(L)$ is embedded in $\operatorname{Po}(K)$. In particular, if $l$ Xh $(K)$, then $\operatorname{Po}(E) \simeq$ $\operatorname{Po}(L)$.

Proof. By Zantema's exact sequence (1.1), $\operatorname{Po}(L)$ is a $2 l$-torsion group. Also $\operatorname{Po}(L)$ is the direct sum of its 2-torsion group $\operatorname{Po}(L)_{2}$ and its $l$-torsion group $\operatorname{Po}(L)_{l}$. By Lemma (2.10), $\epsilon_{L / E}(\operatorname{Po}(E)) \subseteq \operatorname{Po}(L)$. Since $\operatorname{Po}(E)$ is a 2-torsion group,

$$
\left.\mathcal{N}_{L / E} O \epsilon_{L / E}\right|_{\mathrm{Po}(E)}:\left[\Pi_{p}(E)\right] \in \operatorname{Po}(E) \mapsto\left[\Pi_{p}(E)\right]^{l} \in \operatorname{Po}(E)
$$

is injective, and so is $\left.\epsilon_{L / E}\right|_{\operatorname{Po}(E)}: \operatorname{Po}(E) \rightarrow \operatorname{Po}(L)$.

On the other hand, by Proposition (3.1), one can easily show that for an ideal class $\left[\Pi_{p}(L)\right] \in \operatorname{Po}(L)_{2}$, we have $\mathcal{N}_{L / E}\left(\left[\Pi_{p}(L)\right]\right)=\left(\left[\Pi_{p}(E)\right]\right)^{l} \in \operatorname{Po}(E)$. Hence $\mathcal{N}_{L / E}\left(\operatorname{Po}(L)_{2}\right) \subseteq \operatorname{Po}(E)$, and again since $\operatorname{Po}(E)$ is a 2-torsion group,

$$
\left.\mathcal{N}_{L / E}\right|_{\operatorname{Po}(L)_{2}}: \operatorname{Po}(L)_{2} \rightarrow \operatorname{Po}(E)
$$

is injective. Therefore $\operatorname{Po}(E) \simeq \operatorname{Po}(L)_{2}$ and we find the following exact sequence:

$$
\{0\} \longrightarrow \operatorname{Po}(E) \stackrel{\epsilon_{L / E}}{\longrightarrow} \mathrm{Po}(L) \stackrel{\pi_{l}}{\longrightarrow} \mathrm{Po}(L)_{l} \longrightarrow\{0\},
$$

where $\pi_{l}: \operatorname{Po}(L) \rightarrow \operatorname{Po}(L)_{l}$ is the projection map (i.e. multiplication by 2 ).

We claim that $\mathcal{N}_{L / K}\left(\pi_{l}(\operatorname{Po}(L)) \subseteq \operatorname{Po}(K)\right.$ and $\left.\mathcal{N}_{L / K}\right|_{\operatorname{Po}(L)}$ is also injective. To prove these, let $p$ be a ramified prime in $L$ such that $l \mid e(p)$. By Proposition (3.1), $p$ is totally ramified in $K$, say $p \mathcal{O}_{K}=\beta^{l}=\left(\Pi_{p}(K)\right)^{l}$. Two cases are possible:

- If $e(p)=l$ then by Proposition (3.1), one has $p \mathcal{O}_{L}=\left(\gamma_{1} \gamma_{2}\right)^{l}=\left(\Pi_{p}(L)\right)^{l}$ or $p \mathcal{O}_{L}=\gamma^{l}=\left(\Pi_{p^{2}}(L)\right)^{l}$. In this case, depending on whether $f(p)=1$ or $f(p)=2, \mathcal{N}_{L / K}\left(\left[\Pi_{p}(L)\right]\right)=\left[\Pi_{p}(K)\right]^{2} \in \operatorname{Po}(K)$ or $\mathcal{N}_{L / K}\left(\left[\Pi_{p^{2}}(L)\right]\right)=$ $\left[\Pi_{p}(K)\right]^{2} \in \mathrm{Po}(K)$, respectively. Also if $\left(\Pi_{p}(K)\right)^{2}$ is principal, then $\Pi_{p}(K)$ must be principal, since $\left(\Pi_{p}(K)\right)^{l}$ is also principal. On the other hand $j_{L / K}\left(\Pi_{p}(K)\right)=\Pi_{p}(L)$ (resp. $\Pi_{p^{2}}(L)$ ), which implies that $\Pi_{p}(L)$ (resp. $\left.\Pi_{p^{2}}(L)\right)$ is also principal.

- If $e(p)=2 l$, then by Proposition (3.1), $p=l$ ramifies totally in $L$ and so in all its subextensions. In this case $N_{L / K}\left(\Pi_{l}(L)\right)=\Pi_{l}(K)$ and if $\Pi_{l}(K)$ is principal, then $j_{L / K}\left(\Pi_{l}(K)\right)=\left(\Pi_{l}(L)\right)^{2}$ is principal which implies that the ideal class $\left[\Pi_{l}(L)\right]$ belongs to $\operatorname{Po}(L)_{2}$. In other words, $\pi_{l}\left[\Pi_{l}(L)\right] \in \operatorname{Po}(L)_{l} \simeq$ $\frac{\operatorname{Po}(L)}{\epsilon_{L / E}(\operatorname{Po}(E))}$ would be trivial.

Summing up the above arguments, we have proved that

$$
\left.\mathcal{N}_{L / K}\right|_{\mathrm{Po}(L)_{l}}: \operatorname{Po}(L)_{l} \rightarrow \operatorname{Po}(K)
$$

is injective, as claimed. Therefore the following sequence is exact:

$$
\{0\} \longrightarrow \operatorname{Po}(E) \stackrel{\epsilon_{L / E}}{\longrightarrow} \operatorname{Po}(L) \stackrel{\varphi}{\longrightarrow} \operatorname{Po}(K),
$$

where $\varphi:=\left.\mathcal{N}_{L / K}\right|_{\operatorname{Po}(L)_{l}} o \pi_{l}$.

Remark 3.3. Zantema 24], proved that for two finite Galois extensions $K_{1}$ and $K_{2}$ of $\mathbb{Q}$ with $M=K_{1} \cdot K_{2}$, if for every prime number $p$, the ramification indices of $p$ in $K_{1}$ and $K_{2}$ are coprime, then Pólya-ness of $K_{1}$ and $K_{2}$ implies that $M$ is also Pólya. Conversely, if $\operatorname{gcd}\left(\left[K_{1}: \mathbb{Q}\right],\left[K_{2}: \mathbb{Q}\right]\right)=1$ and $M$ is Pólya then $K_{1}$ and $K_{2}$ are Pólya, see [24, Theorem 3.4]. Under these hypotheses, one can 
easily show that $\epsilon_{M / K_{1}}\left(\operatorname{Po}\left(K_{1}\right)\right) \cdot \epsilon_{M / K_{2}}\left(\operatorname{Po}\left(K_{2}\right)\right)=\operatorname{Po}(M)$, see [3]. The condition on relative primality of the degrees is necessary as was shown in [8] and [9] in the case of biquadratic fields. Also the condition on Galois-ness of both $K_{1}$ and $K_{2}$ is necessary:

With the notations in this section, for every ramified prime $p$ in $L$ with $e(p)=2$, by Proposition (3.1), $\Pi_{p}(K)=\beta_{1} \beta_{2} \ldots \beta_{\frac{l+1}{2}}$, and $j_{L / K}\left(\Pi_{p}(K)\right)=\gamma_{1} \Pi_{p}(L)$. Since $\operatorname{Gal}(L / \mathbb{Q})$ acts transitively on the set $\left\{\gamma_{1}, \ldots, \gamma_{l}\right\}, \gamma_{1}$ is not ambiguous. Hence $\epsilon_{L / K}(\mathrm{Po}(K)) \nsubseteq \nsubseteq \mathrm{Po}(L)$.

Corollary 3.4. If all finite places of $E$ are unramified in $L$, then $\operatorname{Po}(L) \simeq \operatorname{Po}(E)$.

Proof. We claim if $L / E$ is unramified, then every ramified prime $p$ in $L / \mathbb{Q}$ has ramification index 2. Otherwise let $p$ be a ramified prime in $L / \mathbb{Q}$ with $l \mid e(p)$, where $e(p)$ denotes the ramification index of $p$ in $L / \mathbb{Q}$. Let $\mathfrak{p}$ and $\gamma$ be prime ideals in $E$ and $L$ above $p$, respectively. Since $l \mid e(p)=e(\gamma / \mathfrak{p}) . e(\mathfrak{p} / p), \mathfrak{p}$ ramifies in $L / E$ and we reach a contradiction. Hence the $l$-torsion subgroup $\operatorname{Po}(L)_{l}$ of $\operatorname{Po}(L)$ would be trivial and the statement follows from Theorem (3.2).

If $L / E$ is unramified, by class field theory $h(E)$ is divisible by $l$. By an argument similar to Honda's result for $l=3$ [11, Proposition 10], we give necessary and sufficient conditions for divisibility of class number of a quadratic field by $l$ :

Lemma 3.5. Suppose that $N$ is a quadratic field and $l \mid h(N)$. Then there exists a $D_{l}$-extension $M$ of $\mathbb{Q}$ such that $N$ is the quadratic subfield of $M$, and $M$ is unramified over $N$. Conversely, let $M$ be a $D_{l}$-extension of $\mathbb{Q}$ which is the splitting field of the irreducible polynomial

$$
f(X)=X^{l}+a_{2} X^{l-2}+a_{3} X^{l-3}+\cdots+a_{l-1} X+a_{l}, \quad a_{i} \in \mathbb{Z}
$$

over $\mathbb{Q}$. If $\operatorname{gcd}\left(a_{2}, a_{3}, \ldots, a_{l-1}\right.$, l. $\left.a_{l}\right)=1$, then class number of the unique quadratic subfield of $M$ is divisible by $l$.

Proof. Assume that $h(N)$ is divisible by $l$. Hence there exists an unramified abelian extension $M$ of degree $l$ over $N$. One can show that $M$ is a Galois extension of $\mathbb{Q}$, thanks to Honda's lemma [11, Lemma 3]. If $\operatorname{Gal}(M / \mathbb{Q}) \simeq C_{2 l}$, the cylic group of order $2 l$, denote the unique subfield of $M$ of degree $l$ over $\mathbb{Q}$ by $F$. Hence $F$ is a cyclic extension of $\mathbb{Q}$, and so every ramified prime $p$ in the extension $F / \mathbb{Q}$, is totally ramified. Therefore, $p$ has the ramification index $l$ or $2 l$ in the extension $M / \mathbb{Q}$. In both cases, there exists a prime ideal of $N$, above $p$ which ramifiies in the extension $M / N$ and we reach a contradiction. Thus $M$ is not abelian over $\mathbb{Q}$, i.e. $\operatorname{Gal}(M / \mathbb{Q}) \simeq D_{l}$.

Conversely, let $M$ be a $D_{l}$-extension of $\mathbb{Q}$. Let $N$ be the unique quadratic subfield of $M$ and denote a subfield of $M$ of degree $l$ over $\mathbb{Q}$ by $F$. Let $\mathfrak{p}$ be a prime ideal of $N$ ramified in $M$. Hence for $p=\mathfrak{p} \cap \mathbb{Q}$, its ramification index is divisible by l. By Proposition (3.1), $p$ totally ramifies in $F / \mathbb{Q}$ which implies that there exists $f(X) \in \mathbb{Z}[X]$ with

$$
f(X) \equiv(X-h)^{l}(\bmod p),
$$

where $h \in \mathbb{Z}$ and $M$ is the splitting field of $f(X)$ over $\mathbb{Q}$. From this congruence equation, we have either

$$
p=l \quad \text { and } \quad l \mid \operatorname{gcd}\left(a_{2}, a_{3}, \ldots, a_{l-1}\right),
$$

or

$$
p \mid \operatorname{gcd}\left(a_{2}, a_{3}, \ldots, a_{l-1}, a_{l}\right) .
$$


Thus $p$ divides $\operatorname{gcd}\left(a_{2}, a_{3}, \ldots, a_{l-1}, l . a_{l}\right)$. On the other hand, if there exists no totally ramified prime in the extension $F / \mathbb{Q}$, by Proposition (3.1) every ramified prime in $M / \mathbb{Q}$ has ramification index 2, which implies that $M / N$ is unramified.

Using Corollary (3.4) and Lemma (3.5), we obtain:

Corollary 3.6. Assume that $L$ is a $D_{l}$-extension of $\mathbb{Q}$ and denote the unique quadratic subfield of $L$ by $E$. Let $L$ be the splitting field of an irreducible polynomial

$$
f(X)=X^{l}+a_{2} X^{l-2}+a_{3} X^{l-3}+\cdots+a_{l-1} X+a_{l}, \quad a_{i} \in \mathbb{Z}
$$

over $\mathbb{Q}$. If $\operatorname{gcd}\left(a_{2}, a_{3}, \ldots, a_{l-1}\right.$, l. $\left.a_{l}\right)=1$, then $\operatorname{Po}(E) \simeq \operatorname{Po}(L)$.

Considering the decomposition form of a prime $p$ in $L$ and $K$, given in Proposition (3.1), we find also a result on divisibilty of class numbers.

Proposition 3.7. 6, Proposition 4.4] If $M / N$ is an extension of number fields, and there is some prime $\mathfrak{p}$ of $\mathcal{O}_{N}$ that is totally ramified in $M / N$, then $h(N) \mid h(M)$.

Corollary 3.8. Let $K$ be a non-Galois number field of degree $l$, for $l$ an odd prime. Let $L$ be the Galois closure of $K$ over $\mathbb{Q}$ with $\operatorname{Gal}(L / \mathbb{Q}) \simeq D_{l}$. Then $h(K) \mid h(L)$. In particular, if $h(L)=1$, then both subfields $K$ and the unique quadratic subfield $E$ of L, are Pólya.

Proof. Let $p$ be a ramified prime in the extension $E / \mathbb{Q}$. Hence $2 \mid e(p)$, where $e(p)$ denotes the ramification index of $p$ in $L / \mathbb{Q}$. If $e(p)=2$, by Proposition (3.1), $p$ has the decomposition form in $K$ as follows:

$$
p \mathcal{O}_{K}=\beta_{1} \beta_{2}^{2} \ldots \beta_{\frac{l+1}{2}}^{2},
$$

which implies that $\beta_{1}$ ramifies in $L / K$. Similarly, if $e(p)=2 l$, then by Proposition (3.1), $p=l$ and in this case the only prime ideal $\beta$ of $K$ above $l$ is ramified in $L / K$. Hence by Proposition (3.7), $h(K) \mid h(L)$. The second assertion follows from Theorem (3.2).

At the end of this section, it might be appropriate to have some numerical examples. To find $D_{5}$-extensions, we use Brumer's generic polynomial (see [13, Definition 0.1.1]):

Proposition 3.9. [13, Theorem 2.3.5] Let $M$ be an arbitrary field. The polynomial (3.6) $f(s, t, X)=X^{5}+(t-3) X^{4}+(s-t+3) X^{3}+\left(t^{2}-t-2 s-1\right) X^{2}+s X+t$ in $M(s, t)[X]$ is then generic for $D_{5}$-extensions over $M$.

Also the quadratic subextension (in chracteristic $\neq 2$ ) of the splitting field of the polynomial $f(s, t, X)$ is obtained by adjoining to $M(s, t)$ a square root of

$$
-\left(4 t^{5}-4 t^{4}-24 s t^{3}-40 t^{3}-s^{2} t^{2}+34 s t^{2}+91 t^{2}+30 s^{2} t+14 s t-4 t-s^{2}+4 s^{3}\right) .
$$

Example 3.10. Consider the notations of Proposition (3.9).

(a) Let $s=5$ and $t=1$ and $K=\mathbb{Q}(\theta)$ where $\theta$ is a root of the polynomial

$$
f(X)=X^{5}-2 X^{4}+7 X^{3}-11 X^{2}+5 X+1 .
$$

We have $D_{K}=1367^{2}$ and $K$ is a $D_{5}$-field. Denote the Galois closure of $K$ over $\mathbb{Q}$ by $L$. By Proposition (3.9),$E=\mathbb{Q}(\sqrt{-1367})$ is the unique quadratic 
subfield of $L$ and by Proposition (1.3), $E$ is Pólya. Since $h(K)=4$, By Theorem (3.2), $L$ is a Pólya $D_{5}$-extension of $\mathbb{Q}$, while by [24, Theorem 6.9] $K$ is not Pólya.

(b) for $s=-5$ and $t=3$ the splitting field $L$ of the polynomial

$$
f(X)=X^{5}-5 X^{3}+15 X^{2}-5 X+3 .
$$

over $\mathbb{Q}$ is a $D_{5}$-extension with the uniuqe quadratic subfield $E=\mathbb{Q}(\sqrt{-15})$. We have $D_{K}=3^{2} .5^{6}, h(K)=1$, where $K=\mathbb{Q}(\theta)$ for some root $\theta$ of $f(X)$. Using Proposition (1.3) and Theorem (3.2), we have $\operatorname{Po}(L) \simeq \operatorname{Po}(E) \simeq C_{2}$.

We recall that for a $D_{l}$-extension $L$ of $\mathbb{Q}$ with quadratic subfield $E$, if $L / E$ is unramified then $\operatorname{Po}(E) \simeq \operatorname{Po}(L)$, see Corollary (3.4).

Proposition 3.11. [14, Section 2] For $s \in \mathbb{Z}$ let

$$
f_{s}(X)=X^{5}-2 X^{4}+(s+2) X^{3}-(2 s+1) X^{2}+s X+1 .
$$

Then, $f_{s}(X)$ is irreducible over $\mathbb{Q}$, $\operatorname{disc}\left(f_{s}(X)\right)=\left(4 s^{3}+28 s^{2}+24 s+47\right)^{2}$ and if $-\left(4 s^{3}+28 s^{2}+24 s+47\right)$ is not a square, then $\operatorname{Gal}(f(X)) \simeq D_{5}$. Moreover, for $\operatorname{Gal}(f(X)) \simeq D_{5}$ the splitting field of $f(X)$ (over $\left.\mathbb{Q}\right)$ is unramified over its unique quadratic subfield, namely over $\mathbb{Q}\left(\sqrt{-4 s^{3}-28 s^{2}-24 s-47}\right)$.

Example 3.12. Let $f_{s}(X)$ be given by equation (3.7). Using Corollary (3.4) one can easily check that the splitting field of $f_{s}(X)$ for $s \in\{ \pm 6, \pm 5, \pm 2, \pm 1,0,8\}$ is a Pólya $D_{5}$-extension of $\mathbb{Q}$, while for $s \in\{ \pm 17, \pm 16, \pm 4,-3,7,9,10\}$ is not.

Following $\left[13\right.$ we give also an example of a Pólya $D_{7}$-extension of $\mathbb{Q}$ :

Example 3.13. Let $K=\mathbb{Q}(\alpha)$ where $\alpha$ is a root of

$$
f(X)=X^{7}-7 X^{6}-7 X^{5}-7 X^{4}-1 .
$$

Then $f(X)$ is irreducible over $\mathbb{Q}$ and the splitting field $L$ of $f(X)$ (over $\mathbb{Q}$ ) has Galois group isomorphic to $D_{7}$, see [13, Section 5.2, Example 5]. Since $\operatorname{disc}(f(X))=$ $-3^{6} .7^{9}, E=\mathbb{Q}(\sqrt{-7})$ is the quadratic subfield of $L$, which is Pólya by Proposition (1.3). Also $h(K)=1$, and by Theorem (3.2) $L$ is Pólya.

Remark 3.14. In [13, Section 7.3], using singular values of certain modular functions, a method for finding dihedral extensions of $\mathbb{Q}$ and some algorithms for constructing the Hilbert class field of an imaginary quadratic field are given. In particular, using Corollary (3.4), one can see that examples (2) and (3) of [13, Section 7.3] are respectively $D_{13}$ and $D_{19}$-extensions of $\mathbb{Q}$. This can also be done by Leriche's result [17, Corollary 3.2].

\section{UPPER BOUND FOR THE NUMBER OF RAMIFICATION}

We again recall that $l$ is an odd prime number. For a number field $M$, denote the number of ramified primes in $M / \mathbb{Q}$ by $s_{M}$. Leriche [16, for any Galois Pólya number field $M$ gave an upper bound for $s_{M}$ which only depends on the degree of $M$ over $\mathbb{Q}$, see [16, Proposition 2.5]. In particular, for a Pólya Galois number field $M$ of degree $2 l$, this upper bound is given by $s_{M} \leq l+4$.

For a cyclic number field $M$ of degree $2 l$, using Zantema's results [24, Proposition 3.2 and Theorem 3.4] the upper bound can be made sharp by $s_{M} \leq 3$.

In [19], we proved that for a non-Galois cubic field $K$ with Galois closure $L$, if $L$ is Pólya depending on whether $D_{K}>0, D_{K}<0$ and $K$ pure, or $D_{K}<0$ and 
$K$ non-pure, then $s_{L} \leq 4, s_{L} \leq 3$ or $s_{L} \leq 2$, respectively. Also by giving some examples, we showed that these upper bounds are actually sharp, see [19, Section $3]$.

In this section, by using the same methods in [19], for a Pólya $D_{l}$-extension $L$ of $\mathbb{Q}$ we give an upper bound for $s_{L}$ which is much smaller than $l+4$.

Theorem 4.1. Let $K$ be a non-Galois number field of prime degree $l>3$. Let $L$ be the Galois closure of $K$ over $\mathbb{Q}$ with $\operatorname{Gal}(L / \mathbb{Q}) \simeq D_{l}$. Denote the unique quadratic subfield of $L$ by $E$, and denote the norm morphism of $L^{\times}$to $K^{\times}$and $E^{\times}$by $N m_{L / K}$ and $N m_{L / E}$, respectively. If $L$ is Pólya, then:

(i) For $L$ real, depending on whether the fundamental unit of $E$ belongs to $N m_{L / E}\left(U_{L}\right)$ or not, $s_{L} \leq 3$ or $s_{L} \leq 4$, respectively.

(ii) For $L$ imaginary, $s_{L} \leq 2$.

Proof. Let $G=\operatorname{Gal}(L / \mathbb{Q}) \simeq D_{l}$. As before, for a ramified prime $p$ in $L$, denote its ramification index by $e(p)$. Since $L$ is a Pólya field, by exact sequence (1.1), we have

$$
\# H^{1}\left(G, U_{L}\right)=\prod_{p \mid D_{L}} e(p),
$$

which implies that $\# H^{1}\left(G, U_{L}\right)$ is a divisor of a power of $2 l$. Now consider the cyclic extensions $L / K$ and $L / E$. Let $G_{2}=\operatorname{Gal}(L / K)$ and $G_{l}=\operatorname{Gal}(L / E)$ and use the Herbrand quotients:

$$
Q\left(G_{2}, U_{L}\right)=\frac{\# \hat{H}^{0}\left(G_{2}, U_{L}\right)}{\# H^{1}\left(G_{2}, U_{L}\right)}, \quad Q\left(G_{l}, U_{L}\right)=\frac{\# \hat{H}^{0}\left(G_{l}, U_{L}\right)}{\# H^{1}\left(G_{l}, U_{L}\right)}
$$

where

$$
\begin{aligned}
\hat{H}^{0}\left(G_{2}, U_{L}\right) & =U_{L}^{G_{2}} / N m_{L / K}\left(U_{L}\right)=U_{K} / N m_{L / K}\left(U_{L}\right), \\
\hat{H}^{0}\left(G_{l}, U_{L}\right) & =U_{L}^{G_{l}} / N m_{L / E}\left(U_{L}\right)=U_{E} / N m_{L / E}\left(U_{L}\right) .
\end{aligned}
$$

On the other hand, the Herbrand quotients $Q\left(G_{2}, U_{L}\right)$ and $Q\left(G_{l}, U_{L}\right)$ are given by [6, Proposition 5.10]:

$$
\begin{aligned}
& Q\left(G_{2}, U_{L}\right)=\frac{2^{s}}{[L: K]}=2^{s-1}, \\
& Q\left(G_{l}, U_{L}\right)=\frac{2^{t}}{[L: E]}=\frac{2^{t}}{l},
\end{aligned}
$$

where $s$ (resp. $t$ ) is the number of infinite places of $K$ (resp. $E$ ) ramified in $L$. For $L$ real (resp. imaginary), the signature of $K$ is $(l, 0)$ (resp. $\left.\left(1, \frac{l-1}{2}\right)\right)$, see 7 , Theorem 9.2.6]. Hence

$$
\begin{aligned}
& Q\left(G_{2}, U_{L}\right)= \begin{cases}\frac{1}{2} & : \text { L is real, } \\
1 & : \text { L is imaginary, }\end{cases} \\
& Q\left(G_{l}, U_{L}\right)=\frac{1}{l} .
\end{aligned}
$$

For the cyclic extension $L / K$, since $N m_{L / K}\left(U_{L}\right)$ contains $U_{K}^{2}$ and $\left(U_{K}: U_{K}^{2}\right)$ divides $2^{w_{K}+1},\left(U_{K}: N m_{L / K}\left(U_{L}\right)\right)$ divides $2^{w_{K}+1}$, where $w_{K}$ denotes the Dirichlet rank of group of units of $K$.

Similarly, for the cylic extension $L / E,\left(U_{E}: N m_{L / E}\left(U_{L}\right)\right)$ divides $l^{w_{E}+1}$. But for $E=\mathbb{Q}(\sqrt{d})$ imaginary, for $d \notin\{-1,-3\}, d=-1$ or $d=-3$ we have $U_{E}=\{ \pm 1\}$, 
$U_{E}=\{ \pm 1, \pm i\}$ or $U_{E}=\left\{ \pm 1, \pm \zeta_{3}, \pm \zeta_{3}^{2}\right\}$, respectively, where $\zeta_{3}=e^{\frac{2 \pi i}{3}}$. Thus for $E$ imaginary, since $\left(U_{E}: N m_{L / E}\left(U_{L}\right)\right)$ divides $\# U_{E}$ and $\operatorname{gcd}\left(\# U_{E}, l\right)=1$, $\left(U_{E}: N m_{L / E}\left(U_{L}\right)\right)=1$.

Now let $E$ be real, and $\xi$ be the fundamental unit of $E$. By Dirichlet Unit Theorem we have $U_{E} \simeq C_{2} \oplus \mathbb{Z}$. Hence depending on whether $\xi \in N m_{L / E}\left(U_{L}\right)$ or not, $\left(U_{E}: N m_{L / E}\left(U_{L}\right)\right)=1$ or $\left(U_{E}: N m_{L / E}\left(U_{L}\right)\right)=l$, respectively.

Summing up the above arguments, and using relations (4.2), (4.3) and (4.4), we find:

- for $L$ real, $\# H^{1}\left(G_{2}, U_{L}\right) \mid 2^{l+1}$ and depending on whether the fundamental unit of $E$ belongs to $N m_{L / E}\left(U_{L}\right)$ or not, $\# H^{1}\left(G_{l}, U_{L}\right)=l$ or $\# H^{1}\left(G_{l}, U_{L}\right)=l^{2}$, respectively.

- for $L$ imaginary, $\# H^{1}\left(G_{2}, U_{L}\right) \mid 2^{\frac{l+1}{2}}$ and $\# H^{1}\left(G_{l}, U_{L}\right)=l$.

On the other hand, the restriction maps

$$
\text { res : } H^{1}\left(G, U_{L}\right) \rightarrow H^{1}\left(G_{2}, U_{L}\right),
$$

and

$$
\text { res : } H^{1}\left(G, U_{L}\right) \rightarrow H^{1}\left(G_{l}, U_{L}\right),
$$

are injective on the 2-primary and $l$-primary part of $H^{1}\left(G, U_{L}\right)$, respectively, see [20, Proposition 1.6.9]. Thus:

$$
\# H^{1}\left(G, U_{L}\right) \mid \# H^{1}\left(G_{2}, U_{L}\right) . \# H^{1}\left(G_{l}, U_{L}\right) \text {. }
$$

Therefore,

- for $L$ real, depending on whether the fundamental unit of $E$ belongs to $N m_{L / E}\left(U_{L}\right)$ or not,

$$
\# H^{1}\left(G, U_{L}\right) \mid 2^{l+1} . l^{1} \quad \text { or } \quad \# H^{1}\left(G, U_{L}\right) \mid 2^{l+1} . l^{2},
$$

respectively.

- for $L$ imaginary,

$$
\# H^{1}\left(G, U_{L}\right) \mid 2^{\frac{l+1}{2}} \cdot l^{1} .
$$

Now since $L$ is Pólya by Theorem (3.2), $E$ is also Pólya. On the other hand, by Proposition (1.3), for real (resp. imaginary) Pólya field $E, s_{E} \leq 2$ (resp. $s_{E}=1$ ). Hence by Theorem (3.2), the 2-torsion subgroup of $\operatorname{Po}(L)$ has at most 2 (resp. 1) cyclic factors. Using the relations (4.6) and (4.7) we find:

- for real Pólya $D_{l}$-extension $L$ of $\mathbb{Q}$, depending on whether the fundamental unit of $E$ belongs to $N m_{L / E}\left(U_{L}\right)$ or not,

$$
\# H^{1}\left(G, U_{L}\right) \mid 2^{2} \cdot l^{1} \quad \text { or } \# H^{1}\left(G, U_{L}\right) \mid 2^{2} \cdot l^{2},
$$

respectively.

- for imaginary Pólya $D_{l}$-extension $L$ of $\mathbb{Q}$,

$$
\# H^{1}\left(G, U_{L}\right) \mid 2^{1} \cdot l^{1} .
$$

Finally using relations (4.1), (4.8) and (4.9), the statement in theorem is proved.

In 12, Ishida proved that for a non-pure number field $M$ of degree $l$, if number of the totally ramified primes in $M / \mathbb{Q}$ is more than the rank of the unit group $w_{M}$, then $l \mid h(M)$, see [12, Theorem 2]. The method used to prove Theorem (4.1), can yield Ishida-type results: 
Corollary 4.2. Let $K$ be a non-Galois cubic field, and denote the number of totally ramified primes in $K / \mathbb{Q}$ by $t_{K}$. Then:

(i) for $D_{K}>0$, if $t_{K} \geq 3$, then $3 \mid \# \operatorname{Po}(K)$;

(ii) for $D_{K}<0$ and $K$ pure, if $t_{K} \geq 3$, then $3 \mid \# \operatorname{Po}(K)$;

(iii) for $D_{K}<0$ and $K$ non-pure, if $t_{K} \geq 2$, then $3 \mid \# \mathrm{Po}(K)$.

In particular, in the above cases $3 \mid h(K)$.

Proof. As before, denote the Galois closure of $K$ over $\mathbb{Q}$ by $L$ and assume that $G=\operatorname{Gal}(L / \mathbb{Q})$. In 19 we proved that

- for $L$ real, $\# H^{1}\left(G, U_{L}\right) \mid 2^{4} .3^{2}$;

- for $L$ imaginary and $K$ pure, $\# H^{1}\left(G, U_{L}\right) \mid 2^{2} .3^{2}$;

- for $L$ imaginary and $K$ non-pure, $\# H^{1}\left(G, U_{L}\right) \mid 2^{2} .3^{1}$;

see [19, proof of Theorem 3.1]. Thereby the 3-primary part of $H^{1}\left(G, U_{L}\right)$ for $L$ real, or $L$ imaginary with $K$ pure has order at most 9 , while for $L$ imaginary with $K$ non-pure has order at most 3 . On the other hand by Zantema's exact sequence (1.1), we have:

$$
\# H^{1}\left(G, U_{L}\right) \cdot \# \operatorname{Po}(L)=\prod_{p \mid D_{L}} e(p) .
$$

(i), (ii). For $L$ real, or $L$ imaginary with $K$ pure, if at least three distinct primes totally ramify in $K / \mathbb{Q}$, then by Proposition (3.1), $3^{3}$ divides $\prod_{p \mid D_{L}} e(p)$. By relation (4.10) and the above arguments, $3 \mid \# \operatorname{Po}(L)$ which implies that the 3 -torsion subgroup $\operatorname{Po}(L)_{3}$ of $\operatorname{Po}(L)$ is nontrivial. By Theorem (3.2), $\operatorname{Po}(L)_{3}$ is embedded in $\operatorname{Po}(K)$.

Part (iii) can be proved similarly.

For $D_{l}$-fields $K$ with $l>3$, one can find a lower bound, independent of $[K: \mathbb{Q}]$, for $t_{K}$ making $h(K)$ divisible by $l$.

Corollary 4.3. Let $K$ be a non-Galois number field of prime degree $l>3$, and assume that the Galois closure of $K$ over $\mathbb{Q}$ has Galois group isomorphic to $D_{l}$. Denote the number of totally ramified primes in $K / \mathbb{Q}$ by $t_{K}$. Then:

(i) for $D_{K}>0$, if $t_{K} \geq 3$, then $l \mid \# \operatorname{Po}(K)$.

(ii) for $D_{K}<0$, if $t_{K} \geq 2$, then $l \mid \# \operatorname{Po}(K)$.

In particular, in the above cases $l \mid h(K)$.

Proof. By an argument similar to proof of Corollary (4.2), and using relations (4.6) and (4.7), the statements are proved.

Remark 4.4. For a $D_{l}$-field $K$ with Galois closure $L$ and unique quadratic subfield $E$ of $L$, one has $D_{K}=\left(D_{E}\right)^{\frac{l-1}{2}} f^{l-1}$, where $f$ is the conductor of $L$ over $E$. Moreover, a prime $p$ is totally ramified in $K / \mathbb{Q}$ if and only if $p \mid f$ and also for $p \mid \operatorname{gcd}\left(D_{E}, f\right)$, we have $p=l$, see [7, Proposition 10.1.28]. Hence, for instance, for a pure cubic field $K=\mathbb{Q}(\sqrt[3]{m})$, where $m=a b^{2}$ with squarefree coprime integers $a>b \geq 1$, if the conductor $f$ of $L=\mathbb{Q}\left(\sqrt[3]{m}, \zeta_{3}\right)$ over $E=\mathbb{Q}(\sqrt{-3})$, has more than two distinct prime divisors, then $3 \mid h(K)$. (Note that $f=a b$ if $m \equiv \pm 1(\bmod 9)$, and $f=3 a b$ otherwise.) 


\section{REFERENCES}

1. A. Brumer, M. Rosen, Class number and ramification in number fields. Nagoya Math. J. 23 (1963), 97-101.

2. P. J. Cahen, J. L. Chabert Integer-valued polynomials. Mathematical Surveys and Monographs 48, Amer. Math. Soc., Providence, 1997.

3. J. L. Chabert, Factorial groups and Pólya groups in Galoisian extensions of $\mathbb{Q}$. Commutative ring theory and applications (Fez 2001), 77-86, Lecture Notes in Pure and Appl. Math., 231, Dekker, New York, 2003.

4. J. L. Chabert, From Pólya Fields to Pólya Groups (I): Galoisian Number Fields. https://arxiv.org/pdf/1805.11585.pdf.

5. J. L. Chabert, From Pólya Fields to Pólya Groups (II): Non-Galoisian Number Fields. https://arxiv.org/pdf/1811.03648.pdf.

6. N. Childress, Class field theory. Springer, New York, 2009.

7. H. Cohen, Advanced topics in computational number theory. Springer-Verlag, New York, 2000.

8. B. Heidaryan, A. Rajaei, Biquadratic Pólya fields with only one quadratic Pólya subfield. J. Number Theory 143 (2014), 279-285.

9. B. Heidaryan, A. Rajaei, Some non-Pólya biquadratic fields with low ramification. Rev. Mat. Iberoam. 33 (2017), 1037-1044.

10. D. Hilbert, The theory of algebraic number fields. (English summary) Translated from the German and with a preface by Iain T. Adamson. With an introduction by Franz Lemmermeyer and Norbert Schappacher. Springer-Verlag, Berlin, 1998.

11. T. Honda, Isogenies, rational points and section points of group varieties. Japan. J. Math. 30 (1960), 84-101.

12. M. Ishida, A note on class numbers of algebraic number fields. J. Number Theory 1 (1969), 65-69.

13. C. U. Jensen, A. Ledet, N. Yui, Generic polynomials. Constructive aspects of the inverse Galois problem. Cambridgre University Press, 2002.

14. M. J. Lavallee, B. K. Spearman, K. S. Williams, Q. Yang, Dihedral quintic fields with a power basis. Math. J. Okayama Univ. 47 (2005), 75-79.

15. A. Leriche, Pólya fields, Pólya groups and Pólya extensions: a question of capitulation. J. Théor. Nombers Bordeaux 23 (2011), 235-249.

16. A. Leriche, Cubic, quartic and sextic Pólya fields. J. Number Theory 133 (2013), 59-71.

17. A. Leriche, About the embedding of a number field in a Pólya field. J. Number Theory 45 (2014), 210-229.

18. A. Maarefparvar, Pólya fields of small degree and low ramification. PhD thesis (in Persian), Tarbiat Modares Univ., December 2017.

19. A. Maarefparvar, A. Rajaei, Pólya $S_{3}$-extensions of $\mathbb{Q}$. Proceedings of the Royal Society of Edinburgh: Section A Mathematics, 1-13. doi:10.1017/prm.2018.86.

20. J. Neukirch, A. Schmidt, K. Wingberg, Cohomology of number fields. Springer-Verlag, Berlin, 2008. 
21. A. Ostrowski, Über ganzwertige Polynome in algebraischen Zahlkörpern. J. Reine Angew. Math.149 (1919), 117-124.

22. G. Pólya, Über ganzwertige Polynome in algebraischen Zahlkörpern. J. Reine Angew. Math. 149 (1919), 97-116.

23. J. P. Serre, Local fields. Translated from the French by Marvin Jay Greenberg. Graduate Texts in Mathematics, 67. Springer-Verlag, New York-Berlin, 1979.

24. H. Zantema, Integer valued polynomials over a number field. Manuscripta Math. 40 (1982), 155-203.

Department of Mathematics, Tarbiat Modares University, 14115-134, Tehran, Iran E-mail address: a.maarefparvar@modares.ac.ir

Department of Mathematics, Tarbiat Modares University, 14115-134, Tehran, Iran

E-mail address: alirajaei@modares.ac.ir 